\title{
RELAXATION METHODS FOR MONOTROPIC PROGRAMS
}

\author{
Paul TSENG* and Dimitri P. BERTSEKAS \\ Laboratory for Information and Decision Systems, Massachusetts Institute of Technology, \\ Cambridge, MA 02139, USA
}

Received 17 September 1987

Revised manuscript received 9 May 1988

\begin{abstract}
We propose a dual descent method for the problem of minimizing a convex, possibly nondifferentiable, separable cost subject to linear constraints. The method has properties reminiscent of the Gauss-Seidel method in numerical analysis and uses the $\varepsilon$-complementary slackness mechanism introduced in Bertsekas, Hosein and Tseng (1987) to ensure finite convergence to near optimality. As special cases we obtain the methods in Bertsekas, Hosein and Tseng (1987) for network flow programs and the methods in Tseng and Bertsekas (1987) for linear programs.
\end{abstract}

Key words: Dual descent, monotropic program, Tucker tableau, elementary vector.

\section{Introduction}

Consider an $n \times m$ real matrix $E$ whose columns are numbered from 1 to $m$ and whose rows are numbered from 1 to $n$. Each column $j$ has associated with it a convex cost function $f_{j}: \mathbb{R} \rightarrow(-\infty,+\infty]$. We consider the problem

$$
\begin{array}{ll}
\text { Minimize } & f(x)=\sum_{j=1}^{m} f_{j}\left(x_{j}\right) \\
\text { subject to } & x \in C,
\end{array}
$$

where $x$ is the vector in $\mathbb{R}^{m}$ with components $x_{j}, j=1,2, \ldots, m$, and $C$ is the subspace

$$
C=\{x \mid E x=0\} .
$$

Note that $f_{j}$ is assumed to be extended real valued so $f_{j}$ can imply interval constraints of the form $l_{j} \leqslant x_{j} \leqslant c_{j}$. In [15], (P) is called a monotropic programming problem. We have assumed that $C$ is a subspace in order to come under the standard framework for monotropic programming, but our algorithm and results can be extended to the case of a linear manifold constraint of the form $C=\{x \mid E x=b\}$, where $b$ is a given vector in $\mathbb{R}^{n}$. We make the following standing assumptions on $f_{j}$.

Work supported by the National Science Foundation under grant NSF-ECS-8519058 and by the Army Research Office under grant DAAL03-86-K-0171.

* This author is also supported by Canadian NSERC under Grant U0505. 
Assumption A. Each $f_{j}$ is lower semicontinuous, and there exists at least one feasible solution for (P), i.e. the effective domain of $f$,

$$
\operatorname{dom}(f)=\{x \mid f(x)<+\infty\}
$$

and $C$ have a nonempty intersection.

Assumption B. The conjugate function [13, p. 104] of $f_{j}$ defined by

$$
g_{j}\left(t_{j}\right)=\sup _{x_{j}}\left\{t_{j} x_{j}-f_{j}\left(x_{j}\right)\right\}
$$

is real valued, i.e. $-\infty<g_{j}\left(t_{j}\right)<+\infty$ for all $t_{j} \in \mathbb{R}$.

Assumption B implies that $f_{j}\left(x_{j}\right)>-\infty$ for all $x_{j}$. It follows that the set of points where $f_{j}$ is real valued, denoted $\operatorname{dom}\left(f_{j}\right)$, is a nonempty interval with right and left endpoints (possibly $+\infty$ or $-\infty$ ) denoted by $c_{j}$ and $l_{j}$, respectively, i.e.

$$
l_{j}=\inf \left\{\xi \mid f_{j}(\xi)<\infty\right\}, \quad c_{j}=\sup \left\{\xi \mid f_{j}(\xi)<\infty\right\} .
$$

It is easily seen that Assumptions A and B imply that for every $t_{j}$ there is some $x_{j} \in \operatorname{dom}\left(f_{j}\right)$ attaining the supremum in (3), and furthermore

$$
\lim _{\left|x_{j}\right| \rightarrow+\infty} f_{j}\left(x_{j}\right)=+\infty \text {. }
$$

It follows that the cost function of $(\mathrm{P})$ has bounded level sets, and therefore (using also the lower semicontinuity of $f$ ) there exists at least one optimal solution to (P).

Rockafellar [15] develops in detail a duality theory, based on Fenchel's duality theorem, involving the dual problem

$$
\begin{array}{ll}
\text { Minimize } & g(t)=\sum_{j=1}^{m} g_{j}\left(t_{j}\right) \\
\text { subject to } & t \in C^{\perp}
\end{array}
$$

where $t$ is the vector with coordinates $t_{j}, j \in\{1,2, \ldots, m\}$, and $C^{\downarrow}$ is the orthogonal complement of $C$,

$$
C^{\perp}=\left\{t \mid t=E^{\mathrm{T}} p \text { for some } p\right\},
$$

where $E^{\mathrm{T}}$ denotes the transpose of the matrix $E$. We will borrow the terminology in [15] for network programming and call an element of $C^{\perp}$ the tension vector. From (5) we have that $t$ is a tension vector if and only if there exists a vector $p$ in $\mathbb{R}^{n}$ such that

$$
t=E^{\mathrm{T}} p .
$$

We will call $p_{i}$, the $i$ th coordinate of $p, i \in\{1,2, \ldots, n\}$, the price of row $i$ of $E$ and we will call $p$ the price vector. Then the dual problem (4) can be written as

(D)

$$
\begin{array}{ll}
\text { Minimize } & q(p) \\
\text { subject to } & \text { no constraint on } p,
\end{array}
$$

where $q$ is the dual functional

$$
q(p)=g\left(E^{\mathrm{T}} p\right) .
$$


Any price vector that attains the minimum in (D) is called an optimal price vector. As shown in [15, Chapter 11D], Assumption A guarantees that there is no duality gap in the sense that the primal and dual optimal costs are negatives of each other.

For each pair $x_{j}$ and $t_{j}$ in $\mathbb{R}$, we say that $x_{j}$ and $t_{j}$ satisfy complementary slackness, CS for short, if

$$
f_{j}^{-}\left(x_{j}\right) \leqslant t_{j} \leqslant f_{j}^{+}\left(x_{j}\right),
$$

where $f_{j}^{-}\left(x_{j}\right)$ and $f_{j}^{+}\left(x_{j}\right)$ denote respectively the left and right derivative of $f_{j}$ at $x_{j}$ (see Figure 1).

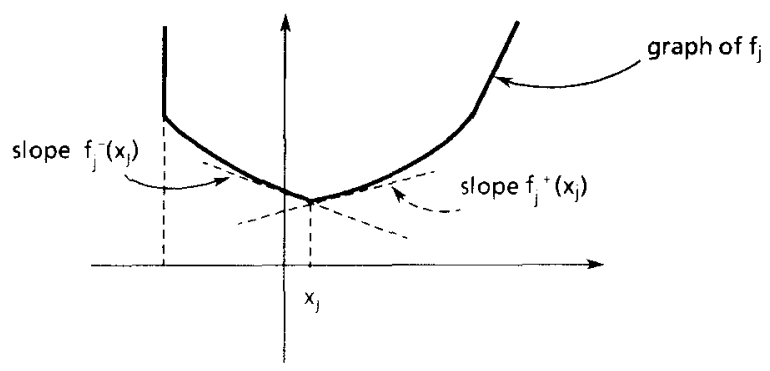

Fig. 1. The left and right derivatives of $f_{j}$ at $x_{j}$.

These derivatives are defined in the usual way for $x_{j}$ in the interior of $\operatorname{dom}\left(f_{j}\right)$. When $-\infty<l_{j}<c_{j}$ we define

$$
f_{j}^{+}\left(l_{j}\right)=\lim _{\xi \downarrow l_{j}} f_{j}^{+}(\xi), \quad f_{j}^{-}\left(l_{j}\right)=-\infty .
$$

When $l_{j}<c_{j}<+\infty$ we define

$$
f_{j}^{-}\left(c_{j}\right)=\lim _{\xi \uparrow c_{j}} f_{j}(\xi), \quad f_{j}^{+}\left(c_{j}\right)=+\infty .
$$

Finally, when $l_{j}=c_{j}$ we define $f_{j}\left(l_{j}\right)=-\infty, f_{j}^{+}\left(c_{j}\right)=+\infty$. We define $g_{j}^{-}\left(t_{j}\right)$ and $g_{j}^{+}\left(t_{j}\right)$ in an analogous manner. Note from the properties of conjugate functions [13] that

$$
g_{j}^{-}(\eta)=\min \left\{\xi \mid f_{j}^{+}(\xi) \geqslant \eta\right\} \quad \text { and } \quad g_{j}^{+}(\eta)=\max \left\{\xi \mid f_{j}(\xi) \leqslant \eta\right\},
$$

which implies that

$$
\lim _{\eta \rightarrow \infty} g_{j}^{-}(\eta)=c_{j} \text { and } \lim _{\eta \rightarrow-\infty} g_{j}^{+}(\eta)=l_{j} .
$$

For each $x$ and $z$ in $\mathbb{R}^{n}$, we denote the directional derivative of $f$ at $x$ in the direction $z$ by

$$
f^{\prime}(x ; z)=\lim _{\mu \downarrow 0} \frac{f(x+\mu z)-f(x)}{\mu} .
$$

Similarly, for each $p$ and $u$ in $\mathbb{R}^{n}$, we denote

$$
q^{\prime}(p ; u)=\lim _{\lambda \downarrow 0} \frac{q(p+\lambda u)-q(p)}{\lambda} .
$$


We will make the following standing assumption in addition to Assumptions A and $\mathrm{B}$.

Assumption C. $f_{j}^{+}\left(l_{j}\right)>-\infty$ for all $j$ such that $l_{j}>-\infty, f_{j}\left(l_{j}\right)<+\infty$, and $f_{j}^{-}\left(c_{j}\right)<+\infty$ for all $j$ such that $c_{j}<+\infty, f_{j}\left(c_{j}\right)<+\infty$.

In the terminology of [15, Chapter 11], Assumption $C$ implies that every feasible primal solution is regularly feasible, and guarantees (together with Assumption A) that the dual problem has an optimal solution [15, Chapter 11].

In this paper we propose a new method for (P) and (D) that in a sense unifies the relaxation methods of $[1,18,20]$ which apply to linear programs, linearly constrained strictly convex cost programs, and convex cost network flow programs, respectively. Our method, which we also call relaxation method, employs the $\varepsilon$-complementary slackness mechanism introduced in [1] and is finitely convergent to within $O(\varepsilon)$ of the optimal cost for any positive $\varepsilon$. We show that this method works with both linear and nonlinear (convex) costs, and contains as special cases the methods in [18] and Section 3 of [1]. If the costs are strictly convex, this method can also work with $\varepsilon=0[1$, Section $2 ; 20]$, in which case it is essentially a dual coordinate descent method. To the best of our knowledge, the only other method for linearly constrained problems with both linear and nonlinear, possibly nondifferentiable and not real valued, costs is Rockafellar's fortified descent method [15, Chapter 11I]. Our method relates in roughly the same way to our linear programming relaxation method [18], as Rockafellar's relates to the classical primal dual method. If $f$ is real valued (not necessarily separable), the active set methods of $[9,11]$ can also be applied. These methods are fundamentally different than ours. Furthermore, to establish convergence, [11] requires the active constraints to be linearly independent at all times and [9] requires the feasible set to be bounded.

The development of this paper proceeds as follows: in Section 2 we introduce the notion of $\varepsilon$-complementary slackness and discuss its relation to dual descent; in Section 3 we review the notion of Tucker tableaus and the painted index algorithm as described in [15, Chapter 10]; in Section 4 we describe the modified version of the painted index algorithm that is used to generate dual descent directions for our method; in Section 5 we present the relaxation method for (P) and (D); in Section 6 we prove finite termination of the method for any positive $\varepsilon$; in Section 7 we show that the cost of the solution generated by the relaxation method is within $O(\varepsilon)$ of the optimal cost and we also show that the dual solution provides useful information about the optimal primal solution.

\section{Dual descent and $\varepsilon$-complementary slackness}

We first introduce some terminology. We will say that a point $b$ in $\operatorname{dom}\left(f_{j}\right)$ is a breakpoint of $f_{j}$ if $f_{j}(b)<f_{j}^{+}(b)$. Note that the dual functional $q$, as given by (7), is 
piecewise either linear or strictly convex. Roughly speaking, each linear piece (breakpoint) of the primal cost function $f_{j}$ corresponds to a breakpoint (linear piece) of the dual cost function $g_{j}$ (see Figure 2).

For a given $\varepsilon \geqslant 0$, we say that $x \in \mathbb{R}^{m}$ and $p \in \mathbb{R}^{n}$ satisfy $\varepsilon$-complementary slackness, $\varepsilon$-CS for short, if

$$
f_{j}^{-}\left(x_{j}\right)-\varepsilon \leqslant t_{j} \leqslant f_{j}^{+}\left(x_{j}\right)+\varepsilon \text { for } j=1,2, \ldots, m,
$$

where $t=E^{\mathrm{T}} p$. For a given $p$, (9) defines upper and lower bounds, called $\varepsilon$-bounds, on the primal variables:

$$
\begin{aligned}
& l_{j}^{\varepsilon}=\min \left\{\xi \mid f_{j}^{+}(\xi) \geqslant t_{j}-\varepsilon\right\}, \\
& c_{j}^{\varepsilon}=\max \left\{\xi \mid f_{j}^{-}(\xi) \leqslant t_{j}+\varepsilon\right\}, \quad \text { for } j=1,2, \ldots, m .
\end{aligned}
$$

Then the $\varepsilon$-CS condition (9) is equivalent to

$$
x_{j} \in\left[l_{j}^{\varepsilon}, c_{j}^{\varepsilon}\right] \text { for } j=1,2, \ldots, m,
$$

where $t=E^{\mathrm{T}} p$. For each $x$ within the $\varepsilon$-bounds, we define the deficit of row $i$ by

$$
d_{i}=\sum_{j=1}^{m} e_{i j} x_{j}
$$

The vector $d$ whose $i$ th coordinate is $d_{i}$ (in vector form $d=E x$ ) is called the deficit vector. Intuition suggests that if $(x, p)$ satisfies $\varepsilon$-CS and $x$ has zero deficit vector (so $x$ is feasible for (P)), then $x$ and $p$ should be near optimal. For a given $t_{j}$, we can obtain $l_{j}^{k}$ and $c_{j}^{*}$ from the characteristic curve of $f_{j}$ graphically (see Figures 3
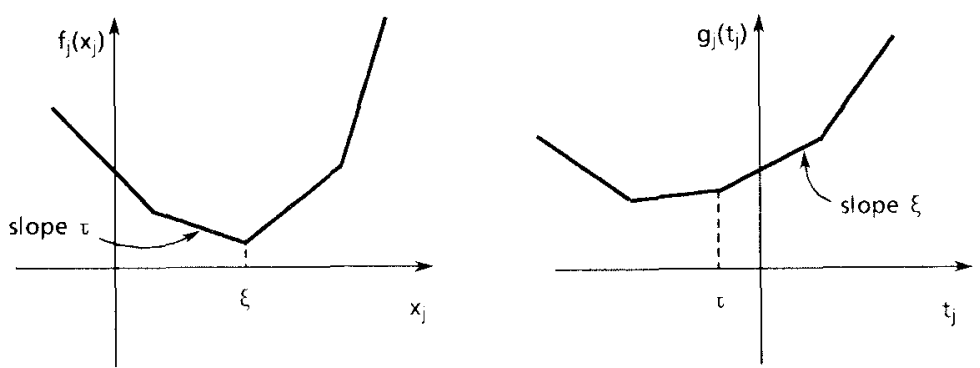

Fig. 2. Correspondence between the breakpoints of $f_{j}$ and the linear pieces of $g_{j}$.

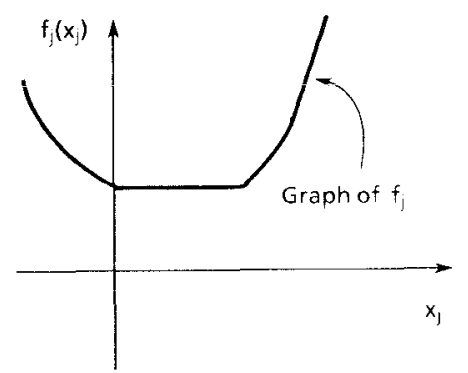

Fig. 3. Graph of $f_{j}$. 


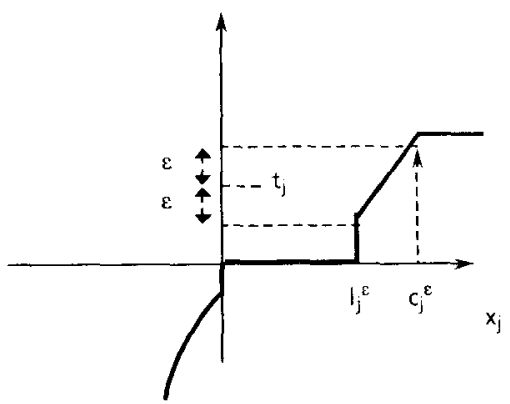

Fig. 4. Graph of $\partial f_{j}$ and $\varepsilon$-bounds corresponding to $t_{j}$.

and 4). The notion of $\varepsilon$-CS and $\varepsilon$-bounds for nonlinear cost has been used in [1] in the context of network flow problems. We apply the same mechanism here to treat the general case where the constraint matrix is not necessarily the node-arc incidence matrix of a directed graph. The definition of $\varepsilon$-CS is related to the $\varepsilon$-subgradient idea used in nondifferentiable optimization [2] as well as to the fortified dual descent method of Rockafellar [15, Chapter 11I], which implements the $\varepsilon$-subgradient method of [2].

It turns out that the $\varepsilon$-bounds can be used to estimate the rate of dual descent. For each tension vector $t$ and vector $v$ in $\mathbb{R}^{m}$ define

$$
C^{\varepsilon}(v, t) \equiv \sum_{v_{j}<0} l_{j}^{\varepsilon} v_{j}+\sum_{v_{j}>0} c_{j}^{\varepsilon} v_{j}
$$

For each $p \in \mathbb{R}^{n}$ and $u \in \mathbb{R}^{n}$, the directional derivative of $q$ at $p$ along $u$ is (cf. (4) and (7)),

$$
q^{\prime}(p ; u)=\sum_{v_{j}<0} g_{j}^{-}\left(t_{j}\right) v_{j}+\sum_{v_{j}>0} g_{j}^{+}\left(t_{j}\right) v_{j},
$$

where $t=E^{\mathrm{T}} p$ and $v=E^{\mathrm{T}} u$. From (10) and [15, Section 8E] we have $g_{j}^{-}\left(t_{j}\right)=l_{j}^{0}$ and $g_{j}^{+}\left(t_{j}\right)=c_{j}^{0}$, so it follows from (13a) that

$$
q^{\prime}(p ; u)=\sum_{v_{j}<0} l_{j}^{0} v_{j}+\sum_{v_{j}>0} c_{j}^{0} v_{j}=C^{0}(v, t)
$$

The following result provides the motivation for using a direction $v$ with $C^{\varepsilon}(v, t)<0$. It shows that such a $v$ leads to descent for all stepsizes not exceeding $\varepsilon /\|v\|$, where $\|\cdot\|$ denotes the sup norm.

Proposition 1. For each price vector $p$ and $u$, if $C^{\varepsilon}(v, t)<0$ then $q^{\prime}(p+\Delta u ; u)<0$ for all $\Delta \in\left[0, \varepsilon^{\prime}\right]$, where $t=E^{\mathrm{T}} p, v=E^{\mathrm{T}} u$ and

$$
\varepsilon^{\prime}=\varepsilon /\|v\|
$$

Proof. We first note from (10) that $l_{j}^{0} \geqslant l_{j}^{\varepsilon}, c_{j}^{0} \leqslant c_{j}^{\varepsilon}$ for all $j$, so we have

$$
C^{0}(v, t)=\sum_{v_{j}<0} l_{j}^{0} v_{j}+\sum_{v_{j}>0} c_{j}^{0} v_{j} \leqslant \sum_{v_{j}<0} l_{j}^{\varepsilon} v_{j}+\sum_{v_{j}>0} c_{j}^{\varepsilon} v_{j}=C^{\varepsilon}(v, t)
$$


Since $q^{\prime}(p ; u)=C^{0}(v, t)$ we obtain $q^{\prime}(p ; u)<0$ so that $u$ is a direction of dual descent at $p$. We will now show that $q^{\prime}\left(p+\varepsilon^{\prime} u ; u\right)<0$. Consider $\left(p^{\prime}, t^{\prime}\right)$ given by

so that

$$
p^{\prime}=p+\varepsilon^{\prime} u, \quad t^{\prime}=t+\varepsilon^{\prime} v
$$

$$
t_{j}^{\prime}=t_{j}+\varepsilon^{\prime} v_{j} \text { for } j=1,2, \ldots, m \text {. }
$$

Then we have (cf. (8a), (10), (14) and (16)),

$$
\begin{aligned}
l_{j}^{e} & \leqslant \min \left\{\xi \mid f_{j}^{+}(\xi) \geqslant t_{j}+\varepsilon v_{j} /\|v\|\right\} \\
& =\min \left\{\xi \mid f_{j}^{+}(\xi) \geqslant t_{j}^{\prime}\right\}=g_{j}^{-}\left(t_{j}^{\prime}\right) \quad \text { for all } j \text { with } v_{j}<0, \\
c_{j}^{\varepsilon} & \geqslant \max \left\{\xi \mid f_{j}^{-}(\xi) \leqslant t_{j}+\varepsilon v_{j} /\|v\|\right\} \\
& =\max \left\{\xi \mid f_{j}^{-}(\xi) \leqslant t_{j}^{\prime}\right\}=g_{j}^{+}\left(t_{j}^{\prime}\right) \quad \text { for all } j \text { with } v_{j}>0 .
\end{aligned}
$$

It follows that

$$
q^{\prime}\left(p+\varepsilon^{\prime} u ; u\right)=\sum_{v_{j}<0} g_{j}^{-}\left(t_{j}^{\prime}\right) v_{j}+\sum_{v_{j}>0} g_{j}^{+}\left(t_{j}^{\prime}\right) v_{j} \leqslant C^{\varepsilon}(v, t),
$$

and therefore $q^{\prime}\left(p+\varepsilon^{\prime} u ; u\right)<0$. Since $q^{\prime}(p ; u)<0$ and $q$ is convex we obtain that $q^{\prime}(p+\Delta u ; u)<0$ for all $\Delta \in\left[0, \varepsilon^{\prime}\right]$.

\section{Tucker tableaus and the painted index algorithm}

In Section 2 we saw (cf. Proposition 1) that if $u$ is such that $C^{x}(v, t)<0$, then $u$ is a dual descent direction along which the line minimization stepsize is "sufficiently large". To generate such $u$ we will use two results from monotropic programming theory: Tucker tableaus and the painted index algorithm [15, Chapter 10]. We will first give a brief overview of Tucker tableaus and then describe the painted index algorithm, which generates, via pivoting, a finite sequence of Tucker tableaus the last of which possesses a special sign pattern.

Consider the linear homogeneous system

$$
T x=0,
$$

where $T$ is a matrix of full row rank. Let each column of $T$ have a unique index. We denote the set of indexes for the columns of $T$ by $J$. Since $T$ has full row rank, we can partition the columns of $T$ into $\left[\begin{array}{ll}T_{\mathrm{B}} & T_{\mathrm{N}}\end{array}\right]$, where $T_{\mathrm{B}}$ is an invertible matrix. Then the equation $T x=0$ can be expressed as

$$
x_{\mathrm{B}}=\left(-T_{\mathrm{B}}^{-1} T_{\mathrm{N}}\right) x_{\mathrm{N}},
$$

where $x=\left(x_{\mathrm{B}}, x_{\mathrm{N}}\right)$. This way of expressing $T x=0$ is a Tucker representation of $S$, where the subspace $S$ is given by $S=\{x \mid T x=0\}$. Similarly,

$$
t_{\mathrm{N}}=\left(T_{\mathrm{B}}^{-1} T_{\mathrm{N}}\right)^{\mathrm{T}} t_{\mathrm{B}},
$$


where $t=\left(t_{\mathrm{B}}, t_{\mathrm{N}}\right)$, is a Tucker representation of $S^{\perp}$, the orthogonal complement of $S$ given by $S^{\perp}=\left\{t \mid t=T^{\mathrm{T}} p\right.$ for some $\left.p\right\}$. The matrix $-T_{\mathrm{B}}^{-1} T_{\mathrm{N}}$ is a Tucker tableau. The columns of $-T_{\mathrm{B}}^{-1} T_{\mathrm{N}}$ are indexed by the indexes of the columns of $T_{\mathrm{N}}$. The rows of $-T_{\mathrm{B}}^{-1} T_{\mathrm{N}}$ are indexed by the indexes of the columns of $T_{\mathrm{B}}$ (see Figure 5). With respect to a given tableau, an index is basic if its corresponding variable is a row variable and nonbasic otherwise. Clearly the number of distinct tableaus is finite.

A nonzero vector $x$ in $S$ is said to be an elementary vector of $S[15$, Chapter 10 ; 12] if there does not exist another nonzero vector $x^{\prime}$ in $S$ such that $\left\{j \mid x_{j}^{\prime}=0\right\}$ strictly contains $\left\{j \mid x_{j}=0\right\}$ (an elementary vector of $S^{\perp}$ is defined analogously). A fundamental relationship exists between the Tucker representations and the elementary vectors of $S$ and $S^{\perp}$ : each column of a Tucker tableau yields in a certain way an elementary vector of $S$, and conversely, each elementary vector of $S$ is obtainable from some column of some Tucker tableau. In a similar way, rows of Tucker tableaus correspond to elementary vectors of the dual subspace $S^{\perp}$. The following is a restatement of the General Basis Theorem [15, p. 457] but using a slightly different normalization.

Proposition 2. For a given Tucker tableau, let $\alpha_{i j}$ denote the entry of the tableau in the row indexed by basic index $i$ and the column indexed by nonbasic index $j$. The elementary vector $z$ of $S$ corresponding to the column indexed by nonbasic $j^{*}$ of the given tableau (normalized so $z_{j^{*}}=1$ ) is

$$
z=\left(\ldots, z_{j}, \ldots\right)_{j \in J}, \quad \text { where } z_{j}= \begin{cases}1 & \text { if } j=j^{*} \\ \alpha_{j j^{*}} & \text { if } j \text { is basic } \\ 0 & \text { else. }\end{cases}
$$

The elementary vector $v$ of $S^{\perp}$ corresponding to the row indexed by basic $i^{*}$ of the given tableau (normalized so $v_{i *}=1$ ) is

$$
v=\left(\ldots, v_{j}, \ldots\right)_{j \in J}, \quad \text { where } v_{j}= \begin{cases}1 & \text { if } j=i^{*} \\ -\alpha_{i{ }^{*} j} & \text { if } j \text { is nonbasic, } \\ 0 & \text { else }\end{cases}
$$

By a painting of the index set $J$ we mean a partitioning of $J$ into four subsets (some possibly empty), whose elements will be called "green", "white", "black", and "red", respectively.

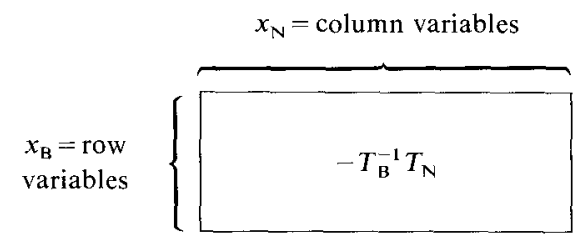

Fig. 5. Tucker tableau corresponding to a partition of $T x=0$ into $T_{\mathrm{B}} x_{\mathrm{B}}+T_{\mathrm{N}} x_{\mathrm{N}}=0$. 


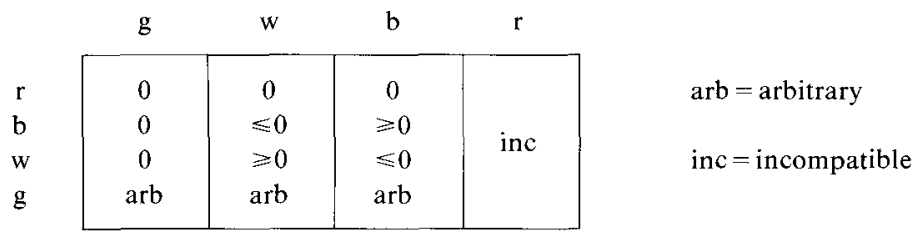

Fig. 6. Column compatibility for Tucker tableau.

\begin{tabular}{|c|c|c|c|c|c|}
\hline & $\mathrm{g}$ & $w$ & b & $\mathrm{r}$ & \multirow{5}{*}{$\begin{array}{l}\text { arb }=\text { arbitrary } \\
\text { inc }=\text { incompatible }\end{array}$} \\
\hline$r$ & 0 & 0 & 0 & arb & \\
\hline b & 0 & $\geqslant 0$ & $\leqslant 0$ & arb & \\
\hline w & 0 & $\leqslant 0$ & $\geqslant 0$ & arb & \\
\hline & \multicolumn{4}{|c|}{ inc } & \\
\hline
\end{tabular}

Fig. 7. Row compatibility for Tucker tableau.

For a given tableau, a column, indexed by say $s$, of the tableau is said to be column compatible if the colour of $s$ and the pattern of signs occurring in that column satisfies the requirements shown in Figure 6. Note that a column whose index is red is never compatible. The requirements for a compatible row are analogously shown in Figure 7.

The painted index algorithm takes any painting of the index set $J$ and any initial Tucker tableau and performs a sequence of pivoting steps to arrive at a final tableau that contains either a compatible column or a compatible row. More explicitly, for any given index $s$ that is black or white, the algorithm produces a final tableau having either a compatible column using $s$ or a compatible row using $s$ (we say that a column (row) uses $s$ if $s$ is either the index of the column (row) or the index of some row (column) whose entry in that column (row) is nonzero). We describe the algorithm below.

Painted index algorithm [15, Chapter 10].

Start with any Tucker tableau. The given white or black index $s$ may correspond to either a row or a column ( $s$ is called the lever index).

If $s$ corresponds to a row, check whether this row is compatible. If yes, we terminate the algorithm. Otherwise there is an entry in the $s$ row that fails the compatibility test. Let $j$ be the index of any column containing such an entry, and check whether this column is compatible. If yes, we terminate the algorithm. Otherwise, there is an entry in column $j$ that fails the compatibility test. Let $k$ be the index of any row containing such an entry. Pivot on $(k, j)$ (i.e. make $j$ basic and $k$ nonbasic) and return to the beginning of the procedure.

If $s$ corresponds to a column, we act analogously to the above, with the word "column" and "row" interchanged. 
The Tucker tableau can be recursively updated after each pivot in a manner similar to that in the simplex method. When the algorithm terminates, either a compatible row using $s$ is found or a compatible column using $s$ is found. The number of distinct Tucker tableaus is finite, thus implying that the number of distinct compatible columns or rows is also finite. To ensure finite termination of the algorithm, Bland's priority rule is suggested: assign priorities to the elements of $J$ arbitrarily and whenever there is more than one index that can be selected as $j$ or $k$, select the one whose priority is highest. We will assume from hereon that this rule is used always.

\section{Generating dual descent directions}

Consider the extended subspace

$$
\Omega=\{(-d, x) \mid d=E x\}
$$

and its orthogonal complement

$$
\Omega^{\perp}=\left\{(p, t) \mid t=E^{\mathrm{T}} p\right\} .
$$

We will describe a particular way to apply the painted index algorithm to determine if a price vector $p$ is approximately dual optimal, and if $p$ is not dual optimal, to either (a) generate an elementary vector $(u, v)$ of $\Omega^{\perp}$ for which $u$ is a dual descent direction of $q$ at $p$, or (b) change the primal vector $x$ so as to reduce the total deficit as measured by

$$
\sum_{i=1}^{n}\left|d_{i}\right|
$$

while maintaining $\varepsilon$-CS with $p$. The scalar $\varepsilon$ is fixed at a positive value throughout the algorithm.

\section{Dual descent generating procedure}

Let $(x, p)$ be a primal dual pair satisfying $\varepsilon$-CS such that $E x \neq 0$. Denote $d=E x$ and select some row $s$ of $E$ with $d_{s} \neq 0$. In the description that follows we assume $d_{s}<0$. The case where $d_{s}>0$ may be treated in an analogous manner.

We apply the painted index algorithm, with $s$ as the lever index, to the extended linear homogeneous system (whose columns are indexed from 1 to $n+m$ ),

$$
\left[\begin{array}{cc}
1,2, \ldots, n & n+1, \ldots, n+m \\
-I & E
\end{array}\right]\left[\begin{array}{l}
w \\
z
\end{array}\right]=0
$$

where index $i$ (corresponding to $w_{i}$ ), $i=1,2, \ldots, n$, is painted

white if $d_{i}>0$,

black if $d_{i}<0$,

red if $d_{i}=0$, 
and index $j+n$ (corresponding to $z_{j}$ ), $j=1,2, \ldots, m$, is painted

$$
\begin{array}{ll}
\text { green } & \text { if } l_{j}^{\varepsilon}<x_{j}<c_{j}^{\varepsilon}, \\
\text { black if } l_{j}^{\varepsilon}=x_{j}<c_{j}^{\varepsilon}, \\
\text { white if } l_{j}^{\varepsilon}<x_{j}=c_{j}^{\varepsilon}, \\
\text { red if } l_{j}^{\varepsilon}=x_{j}=c_{j}^{\varepsilon} .
\end{array}
$$

Furthermore, we (i) use as the initial Tucker tableau one for which $s$ is basic; (ii) assign the lowest priority to index $s$ (this ensures that $s$ is basic during all pivots, as shown in [18, Appendix B]). The key feature of the procedure is that for each Tucker tableau generated by the painted index algorithm we check to see if a dual descent direction can be obtained from its lever row (row indexed by $s$ ):

For each nonbasic index $r$, denote $\alpha_{r}$ the tableau entry in the lever row and the column indexed by $r$. Applying (17b) to the extended linear homogeneous system (20) we obtain the elementary vector $(u, v)$ of $\Omega$ using $s$ that corresponds to the current tableau. This vector is given by

$$
\begin{aligned}
& u_{i}=\left\{\begin{array}{cl}
1 & \text { if } i=s, \\
-\alpha_{i} & \text { if } i \text { is nonbasic, } \\
0 & \text { otherwise, }
\end{array} \quad i=1,2, \ldots, n,\right. \\
& v_{j}= \begin{cases}\alpha_{j+n} & \text { if } j+n \text { is nonbasic, } \\
0 & \text { otherwise, }\end{cases}
\end{aligned}
$$

For this choice of $(u, v)$ we obtain (using (13a), (21) and (22)) that

$$
C^{n}(v, t)=d_{s}-\sum_{i} \alpha_{i} d_{i}+\sum_{\substack{\alpha_{j}+n>0 \\ j+n \text { is not red }}}\left(c_{j}^{z}-x_{j}\right) \alpha_{j+n}+\sum_{\substack{\alpha_{j+n}<0 \\ j+n \text { is not red }}}\left(l_{j}^{v}-x_{j}\right) \alpha_{j+n} .
$$

If $C^{f}(v, t)<0$ then the direction $u$ is a dual descent direction (cf. Proposition 1) and the procedure terminates. Note from (23) that if the tableau is such that the row indexed by $s$ is compatible, then $C^{*}(v, t)<0$ since our choice of index painting and the definition of a compatible row (cf. Figure 7 ) imply that (also using the hypothesis $d_{s}<0$ ),

$$
\begin{aligned}
& d_{s}<0 \quad \text { and } \quad \alpha_{i} d_{i} \geqslant 0 \quad \text { for all } i \text { such that } i \text { is nonbasic; } \\
& x_{j}=c_{j}^{\varepsilon} \text { for all } j \text { such that } j+n \text { is nonbasic and not red, and } \alpha_{j+n}>0 ; \\
& x_{j}=l_{j}^{\varepsilon} \text { for all } j \text { such that } j+n \text { is nonbasic and not red, and } \alpha_{j+n}<0
\end{aligned}
$$

which in view of (21), (22) and (23) implies $C^{\varepsilon}(v, t)<0$.

We know that the painted index algorithm terminates with either a compatible row using $s$ or a compatible column using $s$. Therefore if we do not find a direction $u$ for which $C^{\varepsilon}(v, t)<0$, we must find a compatible column using $s$. In the latter 
case, we compute a direction $z^{*} \in \mathbb{R}^{m}$ from $x$ that decreases the total deficit while maintaining $\varepsilon$-CS:

Denote by $r^{*}$ the index of the compatible column (it can be seen from Figure 6 and the fact that this column uses $s$, that $r^{*}$ is either black or white) and, for each basic index $h$, denote by $\beta_{h}$ the tableau entry in the compatible column and the row indexed by $h$.

Case 1. If $r^{*}=i^{*}$ for some $i^{*} \in\{1, \ldots, n\}$ and $r^{*}$ is black then set

$$
w_{i}^{*} \leftarrow\left\{\begin{array} { l l } 
{ 1 } & { \text { if } i = i ^ { * } , } \\
{ \beta _ { i } } & { \text { if } i \text { is basic, } } \\
{ 0 } & { \text { else, } }
\end{array} \quad z _ { j } ^ { * } \leftarrow \left\{\begin{array}{ll}
\beta_{j+n} & \text { if } n+j \text { is basic, } \\
0 & \text { else. }
\end{array}\right.\right.
$$

Case 2. If $r^{*}=n+j^{*}$ for some $j^{*} \in\{1, \ldots, m\}$ and $r^{*}$ is black then set

$$
w_{i}^{*} \leftarrow\left\{\begin{array} { l l } 
{ \beta _ { i } } & { \text { if } i \text { is basic, } } \\
{ 0 } & { \text { else, } }
\end{array} \quad z _ { j } ^ { * } \leftarrow \left\{\begin{array}{ll}
1 & \text { if } j=j^{*} \\
\beta_{j+n} & \text { if } n+j \text { is basic, } \\
0 & \text { else. }
\end{array}\right.\right.
$$

Case 3. If $r^{*}=i^{*}$ for some $i^{*} \in\{1, \ldots, n\}$ and $r^{*}$ is white then set

$$
w_{i}^{*} \leftarrow\left\{\begin{array} { c l } 
{ - 1 } & { \mathrm { i } i = i ^ { * } , } \\
{ - \beta _ { i } } & { \text { if } i \text { is basic, } } \\
{ 0 } & { \text { else, } }
\end{array} \quad z _ { j } ^ { * } \leftarrow \left\{\begin{array}{cl}
-\beta_{j+n} & \text { if } n+j \text { is basic, } \\
0 & \text { else. }
\end{array}\right.\right.
$$

Case 4. If $r^{*}=n+j^{*}$ for some $j^{*} \in\{1, \ldots, m\}$ and $r^{*}$ is white then set

$$
w_{i}^{*} \leftarrow\left\{\begin{array} { c l } 
{ - \beta _ { i } } & { \text { if } i \text { is basic, } } \\
{ 0 } & { \text { else, } }
\end{array} \quad z _ { j } ^ { * } \leftarrow \left\{\begin{array}{cl}
-1 & \text { if } j=j^{*}, \\
-\beta_{j+n} & \text { if } n+j \text { is basic, } \\
0 & \text { else. }
\end{array}\right.\right.
$$

It can be seen using (17a) and (20) that $w^{*}$ and $z^{*}$ so defined satisfy $w^{*}=E z^{*}$. Furthermore, our choice of index painting, together with column compatibility of the column indexed by $r^{*}$, guarantees that for $\mu>0$ sufficiently small, $x+\mu z^{*}$ satisfies $\varepsilon$-CS with $p$ and $x+\mu z^{*}$ has strictly smaller total deficit than $x$.

Given the above discussion, we see that the modified painted index algorithm will either produce a dual descent direction $u$ as given by (21) that can be used to improve the dual functional, or produce a primal direction $z^{*}$ as given above that can be used to reduce the total deficit.

The special case where the initial tableau is chosen to be $E$ is of particular interest for it leads to the generalized coordinate descent (or relaxation) interpretation of our method. To see this, note that the dual direction $u$ associated with this tableau (cf. (21)) has components

$$
u_{i}= \begin{cases}1 & \text { if } i=s, \\ 0 & \text { otherwise }\end{cases}
$$

so that the coordinate directions are given priority as candidates for dual descent (in fact if the dual cost were differentiable then one could use exclusively such 
coordinate descent directions). Computational tests showed that on linear cost network flow problems the coordinate directions typically contribute between 80 to 90 percent of the total dual cost reduction [17].

\section{Relaxation method}

Based on the discussions in Section 3 and Section 4, we can now formally describe the relaxation method (the scalar $\varepsilon$ is fixed at a positive value throughout the method). The basic relaxation iteration begins with a primal dual pair $(x, p)$ satisfying $\varepsilon$-CS, i.e. $l_{j}^{\varepsilon} \leqslant x_{j} \leqslant c_{j}^{\varepsilon} \forall j$, and returns another pair $\left(x^{\prime}, p^{\prime}\right)$ satisfying $\varepsilon$-CS such that either (i) $q\left(p^{\prime}\right)<q(p)$ or (ii) $p^{\prime}=p$ and (total deficit of $\left.x^{\prime}\right)<($ total deficit of $x$ ).

\section{Relaxation iteration.}

Step 0 . Given $x$ and $p$ satisfying $l_{j}^{\varepsilon} \leqslant x_{j} \leqslant c_{j}^{F} \forall j$, let $t$ and $d$ be the corresponding tension and deficit vectors. If $d=0$ then terminate. Otherwise choose a row $s$ of $E$ for which $d_{s} \neq 0$.

Step 1. Apply the dual descent generating procedure with $s$ as the lever index to the extended linear system

$$
\left[\begin{array}{ll}
-I & E
\end{array}\right]\left[\begin{array}{l}
w \\
z
\end{array}\right]=0
$$

as described in Section 4. If the procedure terminates with a dual descent direction $u$ we go to Step 3. Otherwise the algorithm terminates with a compatible column using $s$, in which case we go to Step 2.

Step 2. (Primal rectification step.) Compute

$$
\mu=\min \left\{\min _{z_{j}^{*}>0} \frac{c_{j}^{r}-x_{j}}{z_{j}^{*}}, \min _{z_{j}^{*}<0} \frac{l_{j}^{k}-x_{j}}{z_{j}^{*}}, \min _{w_{i}^{*} \neq 0} \frac{-d_{i}}{w_{i}^{*}}\right\},
$$

where $z^{*}, w^{*}$ are computed as described in Section 4 . Set

$$
x^{\prime} \leftarrow x+\mu z^{*}, \quad p^{\prime} \leftarrow p,
$$

and exit the iteration. (The choice of $\mu$ above is the largest for which $\varepsilon$-CS is maintained and each deficit is monotonically decreased in magnitude.)

Step 3. (Dual descent step.) Determine a stepsize $\lambda^{*}$ for which

$$
q\left(p+\lambda^{*} u\right)=\min \{q(p+\lambda u) \mid \lambda>0\} .
$$

Set $p^{\prime} \leftarrow p+\lambda^{*} u$ and compute a primal vector $x^{\prime}$ satisfying $\varepsilon$-CS with $p^{\prime}$. Exit the iteration.

\section{Validity and finite termination of the relaxation iteration}

We will show that all steps in the relaxation iteration are executable and that the iteration terminates in a finite number of operations. By the discussion in Section 
4 , the relaxation iteration terminates finitely with either a primal rectification step (Step 2) or a dual descent (Step 3). Step 2 is clearly executable. Step 3 is executable for if there does not exist a line minimization stepsize $\lambda^{*}$ in the direction $u$, then since $u$ is a dual descent direction at $p$, the convexity of $q$ implies

$$
q^{\prime}(p+\lambda u ; u)<0 \quad \forall \lambda>0 .
$$

It follows from (8b) and (13b) that (denoting $v=E^{\mathrm{T}} u$ ) either (i),

$$
\sum_{v_{j}<0} l_{j} v_{j}+\sum_{v_{j}>0} c_{j} v_{j}<0
$$

or (ii),

$$
\sum_{v_{j}<0} l_{j} v_{j}+\sum_{v_{j}>0} c_{j} v_{j}=0
$$

and

either $g_{j}^{-}(\eta)>l_{j} \forall \eta$ for some $j$ with $v_{j}<0$

or $g_{j}^{+}(\eta)<c_{j} \forall \eta$ for some $j$ with $v_{j}>0$.

In case (i), for any $x$ that satisfies $E x=0$ and $l_{j} \leqslant x_{j} \leqslant c_{j}$ for all $j$ (such $x$ exists by Assumption A) we have

$$
0=u^{\mathrm{T}} E x=v^{\mathrm{T}} x=\sum_{v_{j}<0} x_{j} v_{j}+\sum_{v_{j}>0} x_{j} v_{j} \leqslant \sum_{v_{j}<0} l_{j} v_{j}+\sum_{v_{j}>0} c_{j} v_{j}<0,
$$

a clear contradiction. In case (ii), we obtain from (8a) that

$$
\begin{aligned}
& \text { either } f_{j}^{+}\left(l_{j}\right)=-\infty \text { for some } j \text { with } v_{j}<0 \\
& \text { or } f_{j}^{-}\left(c_{j}\right)=+\infty \text { for some } j \text { with } v_{j}>0,
\end{aligned}
$$

and that (using an argument analogous to that for case (i)) any feasible solution $x$ of $(P)$ satisfies

$$
x_{j}=l_{j} \quad \forall j \text { with } v_{j}<0 \quad \text { and } \quad x_{j}=c_{j} \quad \forall j \text { with } v_{j}>0,
$$

which in turn implies

$$
l_{j}>-\infty, \quad f_{j}\left(l_{j}\right)<+\infty, \quad \forall j \text { with } v_{j}<0,
$$

and

$$
c_{j}<+\infty, \quad f_{j}\left(c_{j}\right)<+\infty, \quad \forall j \text { with } v_{j}>0 .
$$

This contradicts Assumption C. 


\section{Finite termination of the relaxation method}

In this section we show that the relaxation method of Section 5 (under a suitable priority assignment of the indexes) terminates in a finite number of iterations. The proof extends the one given in [1] and consists of two parts. In the first part we show that the number of dual descent steps is finite. This is done by arguing that the optimal dual functional is necessarily $-\infty$ if the number of dual descent steps is not finite. In the second part we show that, under an appropriate priority assignment of the indexes, the number of primal rectification steps between successive dual descents is finite.

We first show that the stepsize in each dual descent step is bounded from below by a scalar multiple of $\varepsilon$.

Proposition 3. The stepsize in each dual descent step is at least $\varepsilon / M$, where $M$ is some constant that depends only on $E$.

Proof. For any two price vectors $p$ and $u \in \mathbb{R}^{n}$, Proposition 1 says that if $C^{\varepsilon}(v, t)<0$, where $t=E^{\mathrm{T}} p$ and $v=E^{\mathrm{T}} u$, then $u$ is a dual descent direction at $p+\Delta u$ for all $\Delta \in[0, \varepsilon /\|v\|)$. Therefore the line minimization stepsize is at least $\varepsilon /\|v\|$. Since each dual descent direction $u$ is generated from a Tucker representation of $\Omega^{\perp}$ (cf. (21)) and the number of such representations is finite, we can upper bound $\left\|E^{\mathrm{T}} u\right\|$ from above by the constant

$$
\max \left\{\max \left\{\|v\| \mid \text { all elementary vectors }(u, v) \text { of } \Omega^{\perp} \text { with }\left|u_{s}\right|=1\right\} \mid \text { all } s\right\}
$$

which depends only on $E$.

Denote by $p^{r}$ the price vector generated by the relaxation method just before the $r$ th dual descent step and let $t^{r}=E^{\top} p^{r}$. Also denote by $u^{r}$ the dual descent direction in the price space at the $r$ th dual descent step and let $v^{r}=E^{\mathrm{T}} u^{r}$. We next bound the improvement in the dual functional per dual descent step.

Proposition 4. Let $\varepsilon^{\prime}=\varepsilon / M$ where $M$ is the scalar constant in Proposition 3. Then for each $r \in\{0,1,2, \ldots\}$,

$$
q\left(p^{r}\right)-q\left(p^{r+1}\right)>\sum_{j \text { with } v_{j}^{r} \neq 0}\left[f_{j}\left(\Psi_{j}^{r}\right)-f_{j}\left(\chi_{j}^{r}\right)-\left(\Psi_{j}^{r}-\chi_{j}^{r}\right) t_{j}^{r}\right] \geqslant 0
$$

where we define

$$
\Psi_{j}^{r} \equiv\left\{\begin{array} { l l } 
{ g _ { j } ^ { + } ( t _ { j } ^ { r } + \varepsilon ^ { \prime } v _ { j } ^ { r } ) } & { \text { if } v _ { j } ^ { r } < 0 , } \\
{ g _ { j } ( t _ { j } ^ { r } + \varepsilon ^ { \prime } v _ { j } ^ { r } ) } & { \text { if } v _ { j } ^ { r } > 0 , }
\end{array} \quad \chi _ { j } ^ { r } \equiv \left\{\begin{array}{ll}
g_{j}^{-}\left(t_{j}^{r}\right) & \text { if } v_{j}^{r}<0, \\
g_{j}^{+}\left(t_{j}^{r}\right) & \text { if } v_{j}^{r}>0 .
\end{array}\right.\right.
$$

Proof. From the definition of $\Psi_{j}^{r}$ and $\chi_{j}^{r}$ we have

$$
g_{j}\left(t_{j}^{r}\right)=\chi_{j}^{r} t_{j}^{r}-f_{j}\left(\chi_{j}^{r}\right), \quad g_{j}\left(t_{j}^{r}+\varepsilon^{\prime} v_{j}^{r}\right)=\Psi_{j}^{r}\left(t_{j}^{r}+\varepsilon^{\prime} v_{j}^{r}\right)-f_{j}\left(\Psi_{j}^{r}\right), \quad \forall j \text { with } v_{j}^{r} \neq 0 .
$$


From the definition of $q$ and $v^{r}$ we have

$$
q\left(p^{r}+\varepsilon^{\prime} u^{r}\right)=q\left(p^{r}\right)+\sum_{j \text { with } v_{j}^{r} \neq 0}\left[g_{j}\left(t_{j}^{r}+\varepsilon^{\prime} v_{j}^{r}\right)-g_{j}\left(t_{j}^{r}\right)\right],
$$

and from Proposition 3 we have

$$
q\left(p^{r}\right)-q\left(p^{r+1}\right) \geqslant q\left(p^{r}\right)-q\left(p^{r}+\varepsilon^{\prime} u^{r}\right) .
$$

Combining the above three equalities and inequalities gives

$$
\begin{aligned}
q\left(p^{r}\right)-q\left(p^{r+1}\right) \geqslant & \sum_{j \text { with } v_{j}^{r} \neq 0}\left[\left[\chi_{j}^{r} t_{j}^{r}-f_{j}\left(\chi_{j}^{r}\right)\right]-\left[\Psi_{j}^{r}\left(t_{j}^{r}+\varepsilon^{\prime} v_{j}^{r}\right)-f_{j}\left(\Psi_{j}^{r}\right)\right]\right] \\
= & \sum_{j \text { with } v_{j}^{r} \neq 0}\left[f_{j}\left(\Psi_{j}^{r}\right)-f_{j}\left(\chi_{j}^{r}\right)-\left(\Psi_{j}^{r}-\chi_{j}^{r}\right) t_{j}^{r}\right] \\
& -\varepsilon^{\prime}\left[\sum_{j \text { with } v_{j}^{r} \neq 0} \Psi_{j}^{r} v_{j}^{r}\right] .
\end{aligned}
$$

Since

$$
\begin{aligned}
\sum_{j \text { with } v_{j}^{r} \neq 0} \Psi_{j}^{r} v_{j}^{r} & =\sum_{v_{j}^{r}<0} g_{j}^{+}\left(t_{j}^{r}+\varepsilon^{\prime} v_{j}^{r}\right) v_{j}^{r}+\sum_{v_{j}^{r}>0} g_{j}^{-}\left(t_{j}^{r}+\varepsilon^{\prime} v_{j}^{r}\right) v_{j}^{r} \\
& \leqslant \sum_{v_{j}^{\prime}<0} g_{j}^{-}\left(t_{j}^{r}+\varepsilon^{\prime} v_{j}^{r}\right) v_{j}^{r}+\sum_{v_{j}^{r}>0} g_{j}^{+}\left(t_{j}^{r}+\varepsilon^{\prime} v_{j}^{r}\right) v_{j}^{r} \\
& =q^{\prime}\left(p^{r}+\varepsilon^{\prime} u^{r} ; u^{r}\right)<0,
\end{aligned}
$$

where the last strict inequality holds by Proposition 3, the strict inequality in (24) follows. The right inequality in (24) follows from the convexity of $f_{j}$.

We will now use Propositions 3,4 and Lemma 2 of [20] to prove that the number of dual descent steps is necessarily finite.

Proposition 5. The number of dual descent steps in the relaxation method is finite.

Proof. We will argue by contradiction. Suppose that the number of dual descent steps is infinite. First we show the following property of $\left\{t^{r}\right\}$ : for each $j$,

$$
\begin{aligned}
& \left\{t_{j}^{r}\right\}_{R} \rightarrow \infty \text { for some subsequence } R \Rightarrow c_{j}<+\infty, f_{j}\left(c_{j}\right)<\infty, \\
& \left\{t_{j}^{r}\right\}_{R} \rightarrow-\infty \text { for some subsequence } R \Rightarrow l_{j}>-\infty, f_{j}\left(l_{j}\right)>-\infty .
\end{aligned}
$$

If $\left\{t^{r}\right\}$ is bounded then (25) and (26) trivially hold. Consider a subsequence $R$ for which $\left\{t^{r}\right\}_{R}$ is unbounded. Passing to a subsequence if necessary we assume that, for each component $j,\left\{t_{j}^{r}\right\}_{R}$ is either bounded, or tends to $\infty$, or tends to $-\infty$. By Lemma 2 of [20] there exists $v \in C^{\perp}$ for which $v$ satisfies

$$
v_{j}>0 \quad \text { if } t_{j}^{r} \rightarrow \infty, \quad v_{j}<0 \quad \text { if } t_{j}^{r} \rightarrow-\infty, \quad v_{j}=0 \quad \text { if } t_{j}^{r} \text { is bounded. }
$$

Then for any $\Delta \geqslant 0$,

$$
q^{\prime}\left(p^{r}-\Delta u ;-u\right)=-\sum_{t_{j}^{r} \rightarrow \infty, r \in R} g_{j}^{-}\left(t_{j}^{r}-\Delta v_{j}\right) v_{j}-\sum_{t_{j}^{r} \rightarrow-\infty, r \in R} g_{j}^{+}\left(t_{j}^{r}-\Delta v_{j}\right) v_{j},
$$

and from $(8 \mathrm{~b})$ it follows that

$$
\lim _{r \rightarrow \infty, r \in R} q^{\prime}\left(p^{r}-\Delta u ;-u\right)=-\sum_{v_{j}>0} c_{j} v_{j}-\sum_{v_{j}<0} l_{j} v_{j} .
$$


Let $\theta$ denote the right-hand side of (27). We will argue that $\theta=0$. We cannot have $\theta>0$ since this would imply that there does not exist a primal feasible solution (cf. the argument for case (i) in the validity proof of the relaxation iteration), contradicting Assumption A. We also cannot have $\theta<0$ since then (27) implies that for $r$ sufficiently large

$$
q\left(p^{r}-\Delta u\right) \leqslant q\left(p^{r}\right)+\frac{1}{2} \Delta \theta .
$$

This is not possible since $\Delta$ can be chosen arbitrarily large, while $q(p)$ is bounded from below for all $p$. Thus the only possibility is that $\theta=0$ or

$$
\sum_{v_{j}>0} c_{j} v_{j}+\sum_{v_{j}<0} l_{j} v_{j}=0
$$

Then every feasible primal vector $x$ must satisfy

$$
x_{j}=c_{j} \quad \forall j \text { with }\left\{t_{j}^{r}\right\}_{R} \rightarrow \infty, \quad x_{j}=l_{j} \quad \forall j \text { with }\left\{t_{j}^{r}\right\}_{R} \rightarrow-\infty .
$$

This together with Assumptions A and C imply (25) and (26).

Now we will bound from below the amount of improvement in the dual functional per dual descent by a positive constant. Let $\varepsilon^{\prime}=\varepsilon / M$, where $M$ is the scalar given in Proposition 3. Proposition 3 assures us that at each dual descent step the step length is at least $\varepsilon^{\prime}$. Consider the interval $\left[\frac{1}{4} \varepsilon^{\prime}, \frac{3}{4} \varepsilon^{\prime}\right]$ which we denote by $I$. We have that the dual functional is decreasing on the line segment connecting $p^{r}$ and $p^{r+1}$. It follows from (25), (26) and Assumption $C$ that there exists a subsequence $R$ such that, for each component $j,\left\{t_{j}^{r}\right\}_{R}$ is either bounded, or tends to $\infty$, or tends to $-\infty$; and (also using (13b) and the fact $\left\{v^{r}\right\}$ is bounded since its elements come from a finite set) for $r \in R$ sufficiently large, we have for all $\Delta \in I$,

$$
\begin{aligned}
q^{\prime}\left(p^{r}+\Delta u^{r} ; u^{r}\right)= & \sum_{j \in J^{+}} c_{j} v_{j}^{r}+\sum_{j \in J} l_{j} v_{j}^{r}+\sum_{\substack{j \in J^{0} \\
v_{j}^{\prime}>0}} g_{j}^{+}\left(t_{j}^{r}+\Delta v_{j}^{r}\right) v_{j}^{r} \\
& +\sum_{\substack{j \in J^{\prime} \\
v_{j}^{r}<0}} g_{j}^{-}\left(t_{j}^{r}+\Delta v_{j}^{r}\right) v_{j}^{r}<0,
\end{aligned}
$$

where we define

$$
\begin{aligned}
& J^{+} \equiv\left\{j \mid\left\{t_{j}^{r}\right\}_{r \in R} \rightarrow \infty\right\}, \quad J \cdots\left\{j \mid\left\{t_{j}^{r}\right\}_{r \in R} \rightarrow-\infty\right\}, \\
& J^{0} \equiv\left\{j \mid\left\{t_{j}^{r}\right\}_{r \in R} \text { is bounded }\right\} .
\end{aligned}
$$

Consider a fixed $r \in R$. We define $\theta: \mathbb{R} \rightarrow \mathbb{R}$ by

$$
\theta(\Delta) \equiv q\left(p^{r}+\Delta u^{r}\right)
$$

and we consider two cases. In case (i) the right derivative of $\theta(\Delta)$ assumes at most $2 m$ distinct values in the interval $I$. In case (ii) the right derivative of $\theta(\Delta)$ assumes more than $2 m$ distinct values in the interval $I$. In case (i) $q\left(p^{r}+\Delta u^{r}\right)$ is linear for $\Delta$ in some subinterval of $I^{r}$ of $I$ of length at least $\varepsilon^{\prime} / 4 m$ and it follows from (8a) and $(13 \mathrm{~b})$ that $q^{\prime}\left(p^{r}+\Delta u^{r} ; u^{r}\right)$ over $I^{r}$ is constant of the form

$$
q^{\prime}\left(p^{r}+\Delta u^{r} ; u^{r}\right)=\sum_{j \in J^{+}} c_{j} v_{j}^{r}+\sum_{j \in J^{-}} l_{j} v_{j}^{r}+\sum_{j \in J^{0}} b_{j} v_{j}^{r}
$$


where $b_{j}$ denotes some breakpoint of $f_{j}$ (using the correspondence between the breakpoints of $f_{j}$ and the linear pieces of $g_{j}$ ). This implies that, for each $j \in J^{0}$ such that $v_{j}^{r} \neq 0$, the dual cost $g_{j}\left(t_{j}^{r}+\Delta v_{j}^{r}\right)$ is linear with slope $b_{j}$ for $\Delta$ in $I^{r}$. For each $j \in J^{0},\left\{t_{j}^{r}\right\}_{r \in R}$ is bounded and therefore the number of distinct linear pieces of $g_{j}$ of length $\geqslant \varepsilon^{\prime} / 4 m$ encountered during the course of the method is finite. This together with the fact that $v^{r}$ is chosen from a finite set imply that $q^{\prime}\left(p^{r}+\Delta u^{r} ; u^{r}\right)$ (cf. (29)) can only assume one of a finite set of values over the subinterval $I^{r}$. It follows that in case (i) we can bound the amount of dual cost improvement from below by $\delta \varepsilon / 4 m$, where $\delta$ is some positive scalar. This implies that case (i) can occur for only a finite set of indexes $r$ (for otherwise the dual functional tends to $-\infty$ ) and we need only to consider case (ii). In case (ii), for each $r \in R$ there must exist a $j \in J^{0}$ for which $v_{j}^{r} \neq 0$ and the right derivative of the function $h(\Delta)$ defined by $h(\Delta)=$ $g_{j}\left(t_{j}^{r}+\Delta v_{j}^{r}\right)$ assumes at least three distinct values in the interval $I$. It follows that either $v_{j}^{r}>0$ so that $t_{j}^{r+1} \geqslant t_{j}^{r}+\varepsilon^{\prime} v_{j}^{r}$ and $g_{j}^{+}\left(t_{j}^{r}+\Delta_{1} v_{j}^{r}\right)<g_{j}^{-}\left(t_{j}^{r}+\Delta_{2} v_{j}^{r}\right)$ for at least two points $\Delta_{1}<\Delta_{2}$ in $I$, or $v_{j}^{r}<0$ so that $t_{j}^{r+1} \leqslant t_{j}^{r}+\varepsilon^{\prime} v_{j}^{r}$ and $g_{j}^{+}\left(t_{j}^{r}+\Delta_{2} v_{j}^{r}\right)<g_{j}^{-}\left(t_{j}^{r}+\Delta_{1} v_{j}^{r}\right)$ for at least two points $\Delta_{1}<\Delta_{2}$ in $I$. Passing to a subsequence if necessary we can assume that for all $r \in R, r$ sufficiently large, it is the same $j$ with the preceding property and either $v_{j}^{r}>0$ or $v_{j}^{r}<0$. Without loss of generality we will assume that $v_{j}^{r}>0$ for all $r \in R$ that are sufficiently large. Since $j \in J^{0}$, the subsequence $\left\{t_{j}^{r}\right\}_{r \in R}$ is bounded and therefore has a limit point $t_{j}^{*}$. Passing to a subsequence if necessary, we assume that $\left\{t_{j}^{r}\right\}$ converges to $t_{j}^{*}$. Then it follows that there exists a fixed interval $L$ such that

and

$$
L \subseteq\left(t_{j}^{r}, t_{j}^{r}+\varepsilon^{\prime} v_{j}^{r}\right) \quad \forall r \in R, r \text { sufficiently large, }
$$

$$
\eta_{1}<\eta_{2} \text { and } g_{j}^{+}\left(\eta_{1}\right)<g_{j}^{-}\left(\eta_{2}\right)
$$

for at least two distinct points $\eta_{1}$ and $\eta_{2}$ in $L$. We define

$$
\xi_{1} \equiv g_{j}^{-}\left(\eta_{1}\right), \quad \xi_{2} \equiv g_{j}^{+}\left(\eta_{2}\right)
$$

Then $\xi_{1}$ and $\xi_{2}$ belong to the interval

$$
\left[g_{j}^{-}(a), g_{j}^{+}(b)\right]
$$

where $a, b$ are respectively the left and the right end points of $L$, and (using (8a)) they satisfy

$$
f_{j}^{+}\left(\xi_{1}\right)=\eta_{1}, \quad f_{j}^{-}\left(\xi_{2}\right)=\eta_{2} .
$$

The latter condition together with (31) and the facts $g_{j}^{-}\left(\eta_{1}\right) \leqslant g_{j}^{+}\left(\eta_{1}\right), g_{j}^{--}\left(\eta_{2}\right) \leqslant g_{j}^{+}\left(\eta_{2}\right)$ imply

$$
\xi_{1}<\xi_{2} \text { and } f_{j}^{+}\left(\xi_{1}\right)<f_{j}^{-}\left(\xi_{2}\right) .
$$

Then for $r$ sufficiently large, $r \in R$, we obtain (cf. (30)) that

$$
g_{j}^{+}\left(t_{j}^{r}\right) \leqslant \xi_{1}<\xi_{2} \leqslant g_{j}^{-}\left(t_{j}^{r}+\varepsilon^{\prime} v_{j}^{r}\right)
$$


It follows from Proposition 4 that, for all $r \in R$ that are sufficiently large,

$$
\begin{aligned}
q\left(p^{r}\right)-q\left(p^{r+1}\right) \geqslant & f_{j}\left(g_{j}^{-}\left(t_{j}^{r}+\varepsilon^{\prime} v_{j}^{r}\right)\right)-f_{j}\left(g_{j}^{+}\left(t_{j}^{r}\right)\right) \\
& -f_{j}^{+}\left(g_{j}^{+}\left(t_{j}^{r}\right)\right)\left(g_{j}^{-}\left(t_{j}^{r}+\varepsilon^{\prime} v_{j}^{r}\right)-g_{j}^{+}\left(t_{j}^{r}\right)\right) \\
\geqslant & f_{j}\left(\xi_{2}\right)-f_{j}\left(\xi_{1}\right)-f_{j}^{+}\left(\xi_{1}\right)\left(\xi_{2}-\xi_{1}\right),
\end{aligned}
$$

where the second inequality follows from (32b) and the convexity of $f_{j}$. From (32a) and the convexity of $f_{j}$ we obtain that the right-hand side of (33) is positive. Therefore the dual functional improvement per dual descent is bounded from below by a positive constant, and the dual functional tends to $-\infty$, contradicting Assumption A.

The second part of our finite termination proof consists of showing that if the index priorities are assigned in a certain way, then the number of primal rectification steps between successive dual descents is finite. We have the following:

Proposition 6. If in the relaxation method the green indexes are assigned the highest priorities and the black and white indexes belonging to $\{1,2, \ldots, n\}$, except for the lever index, are assigned the next highest priorities, then the number of primal rectification steps between successive dual descents is finite.

Proof. See [18, Appendix C].

Propositions 5 and 6 imply that the relaxation method of Section 5, using the priority assignment rule stated in Proposition 6, terminates after a finite number of iterations. Since the method only terminates when the deficit vector is the zero vector, the final primal vector $x$ must satisfy $E x=0$. Since $\varepsilon-C S$ is maintained at all relaxation iterations, $x$ and the final price vector must satisfy $\varepsilon$-CS also.

\section{Estimates of suboptimality of solution}

In Section 6 we showed that the relaxation method produces a primal dual pair $(x, p)$ satisfying $\varepsilon$-CS and $x \in C$. In this section we show that the cost of such a pair is within $O(\varepsilon)$ of the optimal cost and furthermore, $p$ yields partial information regarding the optimal primal solution. We begin our argument with the following:

Proposition 7. Let $x \in C$ and $p$ satisfy $\varepsilon-C S$ and let $\xi$ and $p$ satisfy $C S$. Then

$$
0 \leqslant f(x)+q(p) \leqslant \varepsilon \sum_{j=1}^{m}\left|\xi_{j}-x_{j}\right| .
$$


Proof. Let $t=E^{\mathrm{T}} p$. Since $\xi$ and $p$ satisfy CS we have

$$
f_{j}\left(\xi_{j}\right)=t_{j} \xi_{j}-g_{j}\left(t_{j}\right) \quad \forall j=1,2, \ldots, m .
$$

Consider a column $j$ for which $x_{j} \geqslant \xi_{j}$. Then by convexity of $f_{j}$,

$$
f_{j}\left(x_{j}\right)+\left(\xi_{j}-x_{j}\right) f_{j}^{-}\left(x_{j}\right) \leqslant f_{j}\left(\xi_{j}\right)=t_{j} \xi_{j}-g_{j}\left(t_{j}\right)
$$

Hence

$$
f_{j}\left(x_{j}\right)+g_{j}\left(t_{j}\right) \leqslant\left(x_{j}-\xi_{j}\right)\left(f_{j}^{-}\left(x_{j}\right)-t_{j}\right)+t_{j} x_{j} \leqslant\left|\xi_{j}-x_{j}\right| \varepsilon+t_{j} x_{j},
$$

where the second inequality follows from the definition of $\varepsilon$-CS. This inequality is similarly obtained when $x_{j} \leqslant \xi_{j}$ so we have

$$
f_{j}\left(x_{j}\right)+g_{j}\left(t_{j}\right) \leqslant\left|\xi_{j}-x_{j}\right| \varepsilon+t_{j} x_{j} \quad \forall j=1,2, \ldots, m .
$$

From the definition of $g_{j}$ (cf. (3)) we also obtain

$$
t_{j} x_{j} \leqslant f_{j}\left(x_{j}\right)+g_{j}\left(t_{j}\right) \quad \forall j=1,2, \ldots, m .
$$

Summing (34) and (35) over all $j=1,2, \ldots, m$ yields

$$
\sum_{j=1}^{m} t_{j} x_{j} \leqslant \sum_{j=1}^{m}\left[f_{j}\left(x_{j}\right)+g_{j}\left(t_{j}\right)\right] \leqslant \varepsilon \sum_{j=1}^{m}\left|\xi_{j}-x_{j}\right|+\sum_{j=1}^{m} t_{j} x_{j} .
$$

Since $x \in C$ and $t \in C^{\perp}$ we have

$$
\sum_{j=1}^{m} t_{j} x_{j}=0
$$

and the result follows.

Using Proposition 7 we can obtain an explicit bound on the suboptimality of the solution in the special case where $l_{j}>-\infty$ and $c_{j}<+\infty$ for all $j$.

Corollary 1. Suppose $x \in C$ and $p$ satisfy $\varepsilon-C S$. Then

$$
0 \leqslant f(x)+q(p) \leqslant \varepsilon \sum_{j=1}^{m}\left(c_{j}-l_{j}\right)
$$

For the general case we have:

Proposition 8. Let $x(\varepsilon)$ and $p(\varepsilon)$ denote any flow vector and price vector pair for which $x(\varepsilon)$ and $p(\varepsilon)$ satisfy $\varepsilon$-CS and Ex $(\varepsilon)=0$. Then $f(x(\varepsilon))+q(p(\varepsilon)) \rightarrow 0$ as $\varepsilon \rightarrow 0$.

Proof. First we show that $x(\varepsilon)$ remains bounded as $\varepsilon \rightarrow 0$. If $x(\varepsilon)$ is not bounded as $\varepsilon \rightarrow 0$, then since $E x(\varepsilon)=0$ for all $\varepsilon>0$ there exists a sequence $\left\{\varepsilon_{r}\right\} \rightarrow 0$ and a partition of the index set $\{1,2, \ldots, m\}$ into $I^{+}, I^{-}$, and $I^{0}$ such that $c_{j}=+\infty$, 
$x_{j}\left(\varepsilon_{r}\right) \rightarrow+\infty$, for all $j \in I^{+} ; l_{j}=-\infty, x_{j}\left(\varepsilon_{r}\right) \rightarrow-\infty$, for all $j \in I^{-}$; and $x_{j}\left(\varepsilon_{r}\right)$ is bounded for all $j \in I^{0}$. By Assumption B,

$$
\lim _{\xi \rightarrow+\infty} f_{j}^{-}(\xi)=+\infty \quad \text { for all } j \in I^{+}, \quad \lim _{\xi \rightarrow-\infty} f_{j}^{+}(\xi)=-\infty \quad \text { for all } j \in I^{-} .
$$

This and $\varepsilon-\mathrm{CS}$ (cf. (9)) imply

$$
t_{j}\left(\varepsilon_{r}\right) \rightarrow+\infty \text { for all } j \in I^{+}, \quad t_{j}\left(\varepsilon_{j}\right) \rightarrow-\infty \text { for all } j \in I^{-},
$$

where $t\left(\varepsilon_{r}\right)=E^{\mathrm{T}} p\left(\varepsilon_{r}\right)$. Using Lemma 4 in [20] we can express $x\left(\varepsilon_{r}\right)$ as

$$
x\left(\varepsilon_{r}\right)=w^{r}+z^{r} \text { for all } r \text {, }
$$

where $\left\{w^{r}\right\}$ is bounded and $z^{r}$ satisfies

$$
\begin{aligned}
& E z^{r}=0 \forall r \text { and } z_{j}^{r} \rightarrow+\infty \forall j \in I^{+}, \quad z_{j}^{r} \rightarrow-\infty \forall j \in I^{-}, \\
& z_{j}^{r}=0 \quad \forall r \forall j \in I^{0} .
\end{aligned}
$$

Since $t\left(\varepsilon_{r}\right)=E^{\mathrm{T}} p\left(\varepsilon_{r}\right)$, it follows from (37) that

$$
\sum_{j \in I^{+}} t_{j}\left(\varepsilon_{r}\right) z_{j}^{r}+\sum_{j \in I^{-}} t_{j}\left(\varepsilon_{r}\right) z_{j}^{r}=0 \text { for all } r \text {, }
$$

which contradicts (36) and (37). Therefore $x(\varepsilon)$ is bounded as $\varepsilon \rightarrow 0$.

Now we will show that $\xi_{j}(\varepsilon)-x_{j}(\varepsilon)$ is bounded for all $j$ as $\varepsilon \rightarrow 0$, where $\xi(\varepsilon)$ is some vector satisfying $f_{j}^{-}\left(\xi_{j}(\varepsilon)\right) \leqslant t_{j}(\varepsilon) \leqslant f_{j}^{+}\left(\xi_{j}(\varepsilon)\right)$ for all $j$. If $c_{j}<+\infty$ then clearly $\xi_{j}(\varepsilon)$ is bounded from above. If $c_{j}=+\infty$ then by Assumption B, $f_{j}^{-}(\zeta) \rightarrow+\infty$ as $\zeta \rightarrow+\infty$. Then the boundedness of $x_{j}(\varepsilon)$ implies that $t_{j}(\varepsilon)$ is bounded from above, which in turn implies that $\xi_{j}(\varepsilon)$ is bounded from above. Similarly, we can argue that $\xi_{j}(\varepsilon)$ is bounded from below. Therefore $\xi_{j}(\varepsilon)-x_{j}(\varepsilon)$ is bounded for all $j$ as $\varepsilon \rightarrow 0$. This completes our proof in view of Proposition 7 .

Unfortunately Proposition 8 does not tell us a priori how small $\varepsilon$ must be to achieve a certain degree of near optimality. We would need to solve the problem first for some guessed value $\varepsilon$ to obtain $x(\varepsilon)$ and $\xi(\varepsilon)$, evaluate the quality of the solution on the basis of the gap $f(x(\varepsilon))+q(p(\varepsilon))$ between primal and dual solution and then decide whether $\varepsilon$ needs to be decreased. If however the $l_{j}$ 's and the $c_{j}$ 's are finite and known, we can (cf. Corollary 1) obtain an a priori estimate on $\varepsilon$. Nevertheless, the dual solution does yield useful information about the value of the optimal primal solution. This is shown in the following extension of Tardoz's result for linear cost network flow problems [16, Lemma 2.2].

Proposition 9. Let $x^{*}$ denote any optimal primal solution and let $x$ be a primal feasible solution that satisfies $\varepsilon$-CS with some price vector $p$. Let $t=E^{\mathrm{T}} p$. Then

$$
\begin{aligned}
& x_{j}^{*}=l_{j} \quad \forall j \text { with } f_{j}^{+}\left(l_{j}\right)-t_{j}>\varepsilon n M, \\
& x_{j}^{*}=c_{j} \quad \forall j \text { with } f_{j}^{-}\left(c_{j}\right)-t_{j}<-\varepsilon n M,
\end{aligned}
$$

where $M$ is the scalar constant defined by

$$
M \equiv \max _{\substack{B \text { an invertible } \\ \text { submatrix of } E}}\left\{\max _{i, j}\left|\left(B^{-1} E_{B}\right)_{i j}\right|\right\}
$$


and

$$
\begin{aligned}
E_{B}= & \text { submatrix of } E \text { consisting of the rows of } E \text { that correspond } \\
& \text { to the rows of } B .
\end{aligned}
$$

Proof. By making, if necessary, the transformation

$$
\bar{x}_{j} \leftarrow-x_{j}, \quad \bar{l}_{j} \leftarrow-c_{j}, \quad \bar{c}_{j} \leftarrow-l_{j}, \quad \bar{f}_{j}\left(\bar{x}_{j}\right) \leftarrow f_{j}\left(-\bar{x}_{j}\right), \quad \bar{e}_{i j} \leftarrow-e_{i j}
$$

(convexity of the cost function is preserved by this transformation) we can assume that $x \geqslant x^{*}$. We will argue by contradiction. Let $h$ be an index for which

$$
f_{h}^{-}\left(c_{h}\right)-t_{h}<-\varepsilon n M \text { and } x_{h}>x_{h}^{*}
$$

(note that $M \geqslant 1$ so $x_{h}=c_{h}$ by $\varepsilon-\mathrm{CS}$ ). If no such index exists then the claim of the proposition holds since for any $j$ such that $f_{j}^{+}\left(l_{j}\right)-t_{j}>\varepsilon n M$ we have (since $M \geqslant 1$ ) $f_{j}^{+}\left(l_{j}\right)-t_{j}>\varepsilon$, and $\varepsilon$-CS implies that $x_{j}=l_{j}$. On the other hand we have $x_{j} \geqslant x_{j}^{*} \geqslant l_{j}$, so (38) follows.

Let $J \equiv\left\{j \mid x_{j}>x_{j}^{*}\right\}$ and denote $E_{j}$ the $j$ th column of $E$. We note that the set $S \equiv\left\{\xi \mid E \xi=0, \xi_{j}=0 \forall j \not J, \xi_{j} \geqslant 0 \forall j \in J, \xi_{h}>0\right\}$ is nonempty since $x-x^{*}$ belongs to it. Furthermore, for any $\xi$ in $S,\left\{E_{j} \mid j \neq h, \xi_{j}>0\right\}$ is nonempty (otherwise $E_{h}=0$ and (39) cannot hold), and if $\left\{E_{j} \mid j \neq h, \xi_{j}>0\right\}$ does not form a set of linearly independent columns, then it is easily seen that there exists a $\xi^{\prime}$ in $S$ for which $\left\{j \mid \xi_{j}^{\prime}>0\right\} \subsetneq$ $\left\{j \mid \xi_{j}>0\right\}$. It follows that $S$ contains a $\xi$ for which the set of columns $\left\{E_{j} \mid j \neq h, \xi_{j}>0\right\}$ is linearly independent. Let $B$ denote a square submatrix of this set of columns having the same column rank, and let $\xi_{B}$ denote the vector $\left(\ldots, \xi_{j}, \ldots\right)_{j \neq h, \xi_{j}>0}$. It follows that

$$
B \xi_{B}+B_{h} \xi_{h}=0
$$

where $B_{h}$ denotes the portion of $E_{h}$ corresponding to $B$. Then $\xi_{B} / \xi_{h}=-B^{-1} B_{h}$ and from the definition of $M$ we obtain

$$
\sum_{\substack{j \neq h \\ \xi_{i}>0}} \frac{\xi_{j}}{\xi_{h}} \leqslant n M
$$

and that $x^{\prime}=x^{*}+\rho \xi$ is primal feasible, where $\rho \equiv \min \left\{\left(x_{j}-x_{j}^{*}\right) / \xi_{j} \mid j \in J\right\}$. Let $\beta=$ $f\left(x^{\prime}\right)-f\left(x^{*}\right)$. We will show that $\beta<0$, thereby contradicting the optimality of $x^{*}$. Let $K \equiv\left\{j \mid \xi_{j}>0\right\}$. We have

$$
\beta=f\left(x^{\prime}\right)-f\left(x^{*}\right)=\int_{0}^{\rho} f^{\prime}\left(x^{*}+\tau \xi ; \xi\right) \mathrm{d} \tau=\sum_{j \in K} \xi_{j} \int_{0}^{\rho} f_{j}^{+}\left(x_{j}^{*}+\tau \xi_{j}\right) \mathrm{d} \tau .
$$

Since

$$
f_{j}^{+}\left(x_{j}^{*}+\tau \xi_{j}\right) \leqslant f_{j}^{-}\left(x_{j}\right) \quad \forall \tau \in[0, \rho), \forall j \in K,
$$

it follows from (41) that

$$
\beta \leqslant \sum_{j \in K} \xi_{j} \int_{0}^{\rho} f_{j}^{-}\left(x_{j}\right) \mathrm{d} \tau=\sum_{j \in K} \xi_{j} \rho f_{j}^{-}\left(x_{j}\right) .
$$


Since $E \xi=0$, we have that $t^{\mathrm{T}} \xi=0$, or equivalently

$$
-\sum_{j \in K} \xi_{j} t_{j}=0 .
$$

Adding (43) to (42) yields

$$
\beta \leqslant \sum_{j \in K} \xi_{j} \rho\left[f_{j}^{-}\left(x_{j}\right)-t_{j}\right]=\rho \xi_{h}\left[f_{h}^{-}\left(x_{h}\right)-t_{h}\right]+\rho \sum_{j \in K, j \neq h} \xi_{j}\left[f_{j}^{-}\left(x_{j}\right)-t_{j}\right] .
$$

Since (using $\varepsilon$-CS) $f_{j}^{-}\left(x_{j}\right)-t_{j} \leqslant \varepsilon$ for all $j \in K, j \neq h,(40)$ and (44) imply

$$
\beta \leqslant \rho \xi_{h}\left[f_{h}^{-}\left(x_{h}\right)-t_{h}\right]+\rho \varepsilon n M \xi_{h}=\rho \xi_{h}\left[f_{h}^{-}\left(x_{h}\right)-t_{h}+\varepsilon n M\right],
$$

whose right hand side is negative by (39). Thus $\beta<0$ and a contradiction is established.

In general, $M$ is quite difficult to estimate. However, if $E$ is the node-arc incidence matrix for an ordinary network, it can be seen, using the total unimodularity of $E$ [15, p. 135], that $M$ equals one. We can use Proposition 9 to solve (P) and (D) as follows: we apply the relaxation method (with some positive $\varepsilon$ ) to find a feasible primal dual pair satisfying $\varepsilon$-CS, use Proposition 9 to fix a subset of the primal variables at their respective optimal values (which reduces the dimension of the primal vector), and then repeat this procedure with a smaller $\varepsilon$ for the reduced problem. Since the relaxation method converges more rapidly with larger $\varepsilon$ and smaller primal vector dimension, this implementation would be computationally efficient if a large number of primal variables were fixed while $\varepsilon$ is still relatively large (for example when $M$ is small).

\section{Conclusion and extensions}

We have described a dual descent method for monotropic programs. The method uses as descent directions the elementary vectors of a certain extended dual space and, under one particular implementation, has the interpretation of a generalized coordinate descent method. When the $\varepsilon$-complementary slackness mechanism is used, the method is guaranteed to terminate finitely with a feasible primal dual pair whose cost is within $O(\varepsilon)$ of the optimal cost.

A few words concerning practical implementation are in order. The line search in Step 3 can be performed by solving the following single-constraint problem:

$$
\begin{array}{ll}
\text { Minimize } & f(y)-p^{\mathrm{T}} E y \\
\text { subject to } & u^{\mathrm{T}} E y=0 .
\end{array}
$$

When each $f_{j}$ is simple (such as the pointwise maximum of a small number of linear, quadratic and exponential functions) this problem is easily solved. For general $f_{j}$ 's, we can use either procedures analogous to those described in the appendix of [20], or more sophisticated line search procedures such as those described in $[6,8]$. 
Alternatively, it can be seen from Section 6 that exact line search in Step 3 is not crucial for convergence. In particular, any stepsize between $\alpha \varepsilon / M$ and the line search stepsize, where $\alpha$ is a fixed scalar in $(0,1]$ and $M$ is the constant defined in Proposition 3, ensures finite convergence. The bounds $l_{j}^{\varepsilon}$ and $c_{j}^{\varepsilon}$ can also be computed only approximately (for example, any $\xi$ satisfying $\varepsilon \geqslant f_{j}^{+}(\xi)-t_{j} \geqslant \beta \varepsilon$, where $\beta$ is a fixed scalar in $(0,1]$, can be used for $\left.l_{j}^{\varepsilon}\right)$. Furthermore, the value of $\varepsilon$ can be changed after each dual descent step, provided that it remains bounded away from zero. This allows us to perform $\varepsilon$-scaling (i.e. begin with a large $\varepsilon$ and gradually decrease it), which can be beneficial when the cost for different components vary greatly in magnitude.

In the future we hope to code our method to test its practical efficiency. We suspect that it should do well on problems to which second derivative methods are not applicable - as is the case when the costs are linear [3, 18] or piecewise linear/quadratic [1]. It would also be worthwhile to generalize our method either to solve problems whose costs are not separable or to incorporate decomposition techniques to handle problems with side constraints. Some progress has already been made in the latter direction for linear programs [19].

An alternative definition of the $\varepsilon$-bounds that also ensures a finite number of dual descents in the relaxation method is

$$
\sup _{\eta<t_{j}} \frac{g_{j}(\eta)-g_{j}\left(t_{j}\right)+\varepsilon}{\eta-t_{j}}, \quad \inf _{\eta>t_{j}} \frac{g_{j}(\eta)-g_{j}\left(t_{j}\right)+\varepsilon}{\eta-t_{j}}, \quad \text { for } j=1,2, \ldots, m,
$$

used in the fortified dual descent method of Rockafellar ([15], Chapter 11). This alternative definition has the advantage that the cost of the final solution produced is always within $\varepsilon / m$ (as compared to just $O(\varepsilon)$ ) of the optimal cost. However, these $\varepsilon$-bounds appear to be more difficult to compute in practice (for example when the costs are linear).

\section{Acknowledgement}

We are grateful to an anonymous referee for his/her helpful comments.

\section{References}

[1] D.P. Bertsekas, P.A. Hosein and P. Tseng, "Relaxation methods for network flow problems with convex are costs," SIAM Journal on Control and Optimization 25 (1987) 1219-1243.

[2] D.P. Bertsekas and S.K. Mitter, "A descent numerical method for optimization problems with nondifferentiable cost functionals," SIAM Journal on Control 11 (1973) 637-652.

[3] D.P. Bertsekas and P. Tseng, "Relaxation methods for minimum cost ordinary and generalized network flow problems," Operations Research Journal 36 (1988) 93-114.

[4] G.B. Dantzig, Linear Programming and Extensions (Princeton University Press, Princeton, NJ, 1963).

[5] P. Hosein, "Relaxation algorithm for minimum cost network flow problems with convex, separable costs," M.Sc. Thesis in Electrical Engineering and Computer Science, Massachusetts Institute of Technology (Cambridge, MA, 1985). 
[6] C. Lemarechal and R. Mifflin, "Global and superlinear convergence of an algorithm for one dimensional minimization of convex functions," Mathematical Programming 24 (1982) 241-256.

[7] D.G. Luenberger, Introduction to Linear and Nonlinear Programming (Addison-Wesley, Massachusetts, 2nd ed., 1984).

[8] R. Mifflin, "An implementation of an algorithm for univariate minimization and an application to nested optimization," Mathematical Programming Study 31 (1987) 155-166.

[9] V.H. Nguyen and J.J. Strodiot, "A linearly constrained algorithm not requiring derivative continuity," Engineering Structures 6 (1987) 7-11.

[10] J.M. Ortega and W.C. Rheinboldt, Iterative Solution of Nonlinear Equations in Several Variables (Academic Press, New York, 1970).

[11] E.R. Panier, "An active set method for solving linearly constrained nonsmooth optimization problems," Mathematical Programming 37 (1987) 269-292.

[12] R.T. Rockafellar, "The elementary vectors of a subspace of $R^{N}$," in: R.C. Bose and T.A. Dowling, eds., Combinatorial Mathematics and Its Applications (The University of North Carolina Press, Chapel Hill, NC, 1969) pp. 104-127.

[13] R.T. Rockafellar, Convex Analysis (Princeton University Press, Princeton, NJ, 1970).

[14] R.T. Rockafellar, "Monotropic programming: Descent algorithms and duality," in: O.L. Mangasarian, R. Meyer and S. Robinson, eds., Nonlinear Programming, Vol. 4 (Academic Press, New York, 1981) pp. 327-366.

[15] R.T. Rockafellar, Network Flows and Monotropic Programming (Wiley, New York, 1983 ).

[16] E. Tardoz, "A strongly polynomial minimum cost circulation algorithm," Combinatorica 5 (1985) $247-256$.

[17] P. Tseng, "Relaxation method for monotropic programming problems," Ph.D. Thesis, Operations Research Center, Massachusetts Institute of Technology (Cambridge, MA, 1986).

[18] P. Tseng and D.P. Bertsekas, "Relaxation methods for linear programs," Mathematics of Operations Research 12 (1987) 569-596.

[19] P. Tseng and D.P. Bertsekas, "Relaxation methods for linear programs with side constraints," LIDS-P-1696, Laboratory for Information and Decision Systems, Massachusetts Institute of Technology (Cambridge, MA, 1987).

[20] P. Tseng and D.P. Bertsekas, "Relaxation methods for problems with strictly convex separable costs and linear constraints," Mathematical Programming 38 (1987) 303-321. 